Goldschmidt 2021 Abstract

https://doi.org/10.7185/gold2021.3878

\title{
The nanoscale environment of coccolith formation
}

\section{ASSAF GAL}

\section{Weizmann Institute of Science}

Presenting Author: assaf.gal@weizmann.ac.il

The formation of coccoliths, calcium carbonate scales produced by marine unicellular microalgae, is a regulated intracellular process that can dominate the carbon cycle in the marine environment. Traditionally, ion-mediated crystal growth mechanism was primarily used to explain coccolith formation, and to approximate the influence of changing environmental conditions on this biological crystallization process. This view is challenged by observations of numerous competing crystallization pathways in synthetic systems and in other organisms. In this work, we used cryo electron tomography in order to elucidate the cellular nanoscale environment of calcium carbonate coccolith formation. We collected high-resolution 3D information from cells vitrified along the crystallization process. The breadth of data collected allowed us to reconstruct a derived timeline of coccolith development. The native-state imaging of the inorganic phases in their cellular context shows that the crystals nucleate and grow in a confined nanoscale environment, via a complex mechanism that combines both ion-by-ion and particle accretion processes. The nucleation of the crystals is associated with the rim of an organic scale that pattern the ordered array of crystals. In addition, our data show that the membranes engulfing the crystals are molding their shape and leave only minute volume for an aqueous lumen. Such a process emphasizes the sophisticated cellular strategies for controlling biomineralization, and portray that changes in seawater $\mathrm{pH}$ values can affect coccolith formation only indirectly.

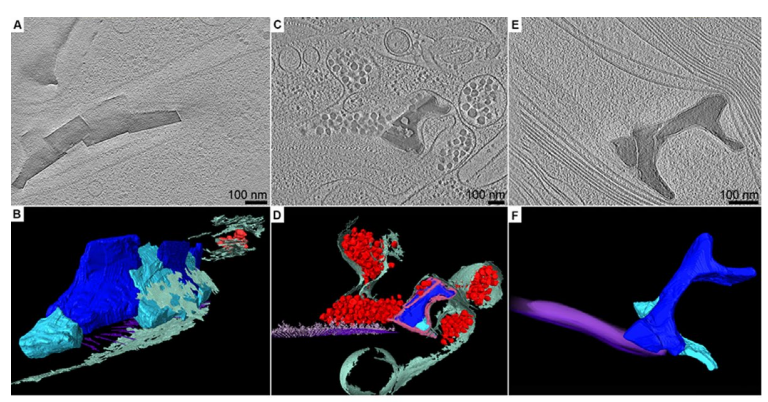

